\title{
372.
}

\section{ON THE RECIPROCATION OF A QUARTIC DEVELOPABLE.}

[From the Quarterly Journal of Pure and Applied Mathematics, vol. vII. (1866), pp. $87-92$.

IT is interesting to consider in a particular case the system of equations which shows $\grave{a}$ posteriori that the reciprocal of a torse (developable surface) is a curve (curve in space); and the reciprocal system which shows that the reciprocal of a curve is a torse.

Using $(a, b, c, d)$ and $(x, y, z, w)$ for the reciprocal coordinates, it will be convenient to collect the different equations as follows:

$$
\begin{aligned}
& a^{2} d^{2}-6 a b c d+4 a c^{3}+4 b^{3} d-3 b^{2} c^{2}=0, \\
& a d^{2}-3 b c d+2 c^{3}+\lambda x=0, \\
& -3 a c d+6 b^{2} d-3 b c^{2}+\lambda y=0 \text {, } \\
& -3 a b d+6 a c^{2}-3 b^{2} c-\lambda z=0 \text {, } \\
& a^{2} d-3 a b c+2 b^{3}+\lambda w=0, \\
& a x+b y+c z+d w=0, \\
& 3 x z-y^{2}=0, \quad y z-9 x w=0, \quad 3 y w-z^{2}=0, \\
& 3 p z-9 q w \quad+a=0, \\
& -2 p y+q z+3 r w+b=0 \text {, } \\
& 3 p x+q y-2 r z+c=0 \text {, } \\
& -9 q x+3 r y+d=0,
\end{aligned}
$$

c. V. 
This being so, the equation (1) belongs to a quartic torse, the reciprocal whereof is the skew cubic determined by the equations (4): and we have to show $\grave{a}$ posteriori that this is so.

First if the torse is given, then the reciprocal figure is the envelope of the plane $a x+b y+c z+d w=0,(3)$, where $(x, y, z, w)$ are the coordinates and $(a, b, c, d)$ are regarded as variable parameters connected by the equation (1); we thence obtain the equations (2), where $\lambda$ is an arbitrary multiplier; and from the equations (1), (2), and (3), we have to eliminate $a, b, c, d, \lambda$. The equation (3) is at once seen to be included in the equations (1) and (2); and the elimination would give only a single equation between the $(x, y, z, w)$-since however the equation (1) is that of a torse, we know that the elimination must give two equations, or more accurately a two-fold relation (represented, as in the present case it happens, by three equations) between the coordinates $(x, y, z, w)$.

Putting for shortness

$$
=a^{2} d^{2}-6 a b c d+4 a c^{3}+4 b^{3} d-3 b^{2} c^{2}
$$

and substituting for $\lambda x, \lambda y, \lambda z, \lambda w$, their values from the equations (1), we have identically

$$
\begin{aligned}
& \lambda^{2}\left(x z-\frac{1}{3} y^{2}\right)=-\left(b d-c^{2}\right) \square \\
& \lambda^{2}\left(\frac{1}{9} y z-x w\right)=-(a d-b c) \square \\
& \lambda^{2}\left(y w-\frac{1}{3} z^{2}\right)=-\left(a c-b^{2}\right) \square ;
\end{aligned}
$$

and hence (since $\square=0$ ) we have between $(x, y, z, w)$ the equations (4), showing that the reciprocal figure is a skew cubic.

Secondly, let the skew cubic be given; then the reciprocal figure is the envelope of the plane $a x+b y+c z+d w=0$, (3), where now $(a, b, c, d)$ are the coordinates and $(x, y, z, w)$ are regarded as variable parameters connected by the equations (4): we thence obtain the equations (5) containing the arbitrary multipliers $p, q, r$ : and from the equations (3), (4), (5) we have to eliminate $x, y, z, w, p, q, r$. The equation (3) is at once seen to be included in the equations (4) and (5): since however the equations (4) are those of a curve, we know that the elimination must give a single equation between $(a, b, c, d)$.

The equations (4) are satisfied if we write therein

$$
x: y: z: w=\frac{1}{3}: \theta: \theta^{2}: \frac{1}{3} \theta^{3},
$$

and substituting these values of $x, y, z, w$ in the equations ( 5$)$, these equations give $(a, b, c, d)$ in terms of $\theta, p, q, r$, and we thence find identically

that is

$$
\begin{aligned}
& a c-b^{2}=-\theta^{2}\left(p-2 q \theta+r \theta^{2}\right)^{2}, \\
& b c-a d=-2 \theta(p-2 q \theta+r \theta)^{2}, \\
& b d-c^{2}=-\quad\left(p-2 q \theta+r \theta^{2}\right)^{2},
\end{aligned}
$$

$$
a c-b^{2}: a d-b c: b d-c^{2}=\theta^{2}:-2 \theta: 1,
$$


or we have

$$
(a d-b c)^{2}-4\left(a c-b^{2}\right)\left(b d-c^{2}\right)=0,
$$

which is in fact the equation

$$
\square=a^{2} d^{2}-6 a b c d+4 a c^{3}+4 b^{3} d-3 b^{2} c^{2}=0,
$$

and thus the reciprocal is in fact the quartic torse, given by the equation (1).

The equations (3), (4), (5) lead to

$$
x \delta a+y \delta b+z \delta c+w \delta d=0 ;
$$

but $(a, b, c, d)$ are in consequence of these equations connected by a single equation, viz. the equation (1); the equation just obtained is thus the only relation between the differentials $(\delta a, \delta b, \delta c, \delta d)$ : it is clear from the equations (2) that this is in fact the equation

$$
\delta \square=\delta_{a} \square \delta a+\delta_{b} \square \delta b+\delta_{c} \square \delta c+\delta_{d} \square \delta d=0 .
$$

The equations (1), (2), (3) lead in like manner to

$$
a \delta x+b \delta y+c \delta z+d \delta w=0
$$

which in virtue of the equations ( $\check{)}$ is equivalent to

$$
p \delta\left(3 x z-y^{2}\right)+q \delta(y z-9 x w)+r \delta\left(3 y w-z^{2}\right)=0
$$

viz. it is a consequence of

$$
\delta\left(3 x z-y^{2}\right)=0, \quad \delta(y z-9 x w)=0, \quad \delta\left(3 y w-z^{2}\right)=0 .
$$

But inasmuch as $(x, y, z, w)$ are connected by a two-fold relation, the equations (1), (2), (3) must lead to one other linear relation between the differentials $(\delta x, \delta y, \delta z, \delta w)$ : and I proceed to show that this is so.

Differentiating we have

$$
\begin{aligned}
& d^{2} \delta \dot{a}: \quad-3 c d \delta b+\left(-3 b d+6 c^{2}\right) \delta c+(2 a d-3 b c) \delta d+\lambda d x+x \delta \lambda=0, \\
& -3 c d \delta a+\left(12 b d-3 c^{2}\right) \delta b+(-3 a d-6 b c) \delta c+\left(-3 a c-6 b^{2}\right) \delta d+\lambda d y+y d \lambda=0, \\
& \left(-3 b d+6 c^{2}\right) \delta a+(-3 a d-6 b c) \delta b+\left(12 a c-3 b^{2}\right) \delta c-\quad 3 a b \delta d+\lambda d z+z d \lambda=0, \\
& (2 a d-3 b c) \delta a+\left(-3 a c+6 b^{2}\right) \delta b-\quad 3 a b \delta c+\quad a^{2} \delta d+\lambda d w+w d \lambda=0 .
\end{aligned}
$$

Consider the matrix

$$
\begin{aligned}
& d^{2} \quad, \quad-3 c d \quad, \quad-3 b d+6 c^{2}, \quad 2 a d-3 b c \\
& -3 c d \quad, \quad 12 b d-3 c^{2}, \quad-3 a d-6 b c,-3 a c+6 b^{2} \\
& -3 b d+6 c^{2}, \quad-3 a d-6 b c, \quad 12 a c-3 b^{2}, \quad-3 a b \\
& 2 a d-3 b c,-3 a c+6 b^{2},-3 a b \quad, \quad a^{2}
\end{aligned}
$$


and suppose for a moment that the determinant formed therewith is $=K$; suppose also that the reciprocal matrix is

$$
\left|\begin{array}{llll}
\mathfrak{H}, & \mathfrak{H}, & \mathfrak{S}, & \mathfrak{R} \\
\mathfrak{S}, & \mathfrak{B}, & \mathfrak{F}, & \mathfrak{N} \\
\mathfrak{S}, & \mathfrak{F}, & \mathfrak{E}, & \mathfrak{N} \\
\mathfrak{R}, & \mathfrak{M}, & \mathfrak{N}, & \mathfrak{D}
\end{array}\right|
$$

Then we have

$$
K \delta a+d \lambda(\mathfrak{2} x+\mathfrak{S} y+\mathfrak{d} z+\mathfrak{Q} w)+\lambda(\mathfrak{2} d x+\mathfrak{S} d y+\mathfrak{d} d z+\mathfrak{Q} d w)=0,
$$

with the similar equations involving $b, c, d$ and $(\mathfrak{S}, \mathfrak{B}, \mathfrak{F}, \mathfrak{M}),(\mathfrak{S}, \mathfrak{F}, \mathfrak{(}, \mathfrak{R}),(\mathfrak{R}, \mathfrak{M}, \mathfrak{N}, \mathfrak{D})$ respectively.

But, substituting for $(x, y, z, w)$ their values from the equations (2), it is easy to see that we have

$$
\mathfrak{A} x+\mathfrak{S} y+\mathfrak{S} z+\mathfrak{Q} w=-\frac{1}{3 \lambda} K a,
$$

the last-mentioned equation thus is

$$
K\left(\delta a-\frac{1}{3} \frac{a}{\lambda} \delta \lambda\right)+\lambda(\mathfrak{2} \delta x+\mathfrak{S} \delta y+\mathscr{S} \delta z+\mathfrak{\Omega} \delta w)=0 .
$$

But $K$ is $=27 \square$ (see my paper "On Certain Developable Surfaces," Quarterly Mathematical Journal, t. vi. 1864, pp. 108-126, [3444]), which is $=0$, and we thus have

and similarly

$$
\mathfrak{2} \delta x+\mathfrak{S} \delta y+\mathfrak{C} \delta z+\mathfrak{Q} \delta w=0,
$$

$$
\begin{aligned}
& \mathfrak{S} \delta x+\mathfrak{B} \delta y+\mathfrak{F} \delta z+\mathfrak{M} \delta w=0, \\
& \mathfrak{S} \delta x+\mathfrak{\mho} \delta y+(\overleftarrow{ } \delta z+\mathfrak{N} \delta w=0, \\
& \mathfrak{R} \delta x+\mathfrak{M} \delta y+\mathfrak{R} \delta z+\mathfrak{D} \delta w=0 .
\end{aligned}
$$

But observing that in virtue of the equation $K=0$, we have

$$
\mathfrak{A}: \mathfrak{H}: \mathfrak{F}: \mathfrak{R}=\mathfrak{H}: \mathfrak{B}: \mathfrak{F}: \mathfrak{M}=\mathfrak{H}: \mathfrak{F}:(\mathfrak{S}: \mathfrak{R}=\mathfrak{R}: \mathfrak{M}: \mathfrak{R}: \mathfrak{D} \text {, }
$$

these are, as they should be, one and the same equation.

The values of the coefficients $\mathfrak{A}, \mathfrak{B}, \mathbb{\&}$ c. are given, p. 112 of the paper just referred to, viz. writing $\square$ in place of $U$, we have $\mathfrak{A}=3 X^{2}-4 a^{2} \square$, \&c. where

$$
\begin{aligned}
& X=a^{2} d-3 a b c+2 b^{3}, \\
& Y=a b d-2 a c^{2}+b^{2} c, \\
& Z=-a c d+2 b^{2} d-b c^{2}, \\
& W=-a d^{2}+3 b c d-2 c^{2}
\end{aligned}
$$


and writing $\square=0$, the values each divided by 3, are simply

$$
\begin{aligned}
& X^{2}, X Y, X Z, X W, \\
& Y X, Y^{2}, Y Z, Y W, \\
& Z X, Z Y, Z^{2}, Z W \text {, } \\
& W X, W Y, W Z, W^{2} \text {, }
\end{aligned}
$$

so that each of the four equations in fact, becomes

$$
X \delta x+Y \delta y+Z \delta z+W \delta w=0,
$$

or multiplying by 3, and attending to the equations (2), this is

$$
-3 w \delta x+z \delta y-y \delta z+3 x \delta w=0 .
$$

This should be a consequence of the equations

$$
\delta\left(3 x z-y^{2}\right)=0, \quad \delta(y z-9 x w)=0, \quad \delta\left(3 y w-z^{2}\right)=0,
$$

that is, we should be able from the first three to deduce the fourth equation in the system

$$
\begin{array}{r}
3 z \delta x-2 y \delta y+3 x \delta z=0 \\
-9 w \delta x+z \delta y+y \delta z-9 x \delta w=0 \\
3 w \delta y-2 z \delta z+3 y \delta w=0 \\
-3 w \delta x+z \delta y-y \delta z+3 x \delta w=0
\end{array}
$$

or we ought to have

$$
\left|\begin{array}{rrrr}
3 z, & -2 y, & 3 x, & \\
-9 w, & z, & y, & -9 x \\
& 3 w, & -2 z, & 3 y \\
-3 w, & z, & -y, & 3 x
\end{array}\right|=0
$$

but expanding, this is

or.

$$
-6\left(81 x^{2} w^{2}-54 x y z w+12 x z^{3}+12 y^{3} w-3 y^{2} z^{2}\right)=0,
$$

$$
(y z-9 x w)^{2}-4\left(x z-y^{2}\right)\left(y w-z^{2}\right)=0
$$

which is true in virtue of the relations (4). Or what is the same thing, we may show without difficulty that the equation

$$
-3 w \delta x+z \delta y-y \delta z+3 x \delta w=0,
$$

is satisfied by writing therein $x: y: z: w=\frac{1}{3}: \theta: \theta^{2}: \frac{1}{3} \theta^{3}$. 
I remark that in general, if $\square=\phi(a, b, c, d)=0$ is the equation of a torse, then for finding the reciprocal curve, we have

$$
\begin{array}{ll}
\square=0, & a x+b y+c z+d w=0, \\
& \delta_{a} \square+\lambda x=0, \\
& \delta_{b} \square+\lambda y=0, \\
& \delta_{c} \square+\lambda z=0, \\
& \delta_{d} \square+\lambda w=0,
\end{array}
$$

and that from these equations we deduce not only

$$
a \delta x+b \delta y+c \delta z+d \delta w=0
$$

but also the equation

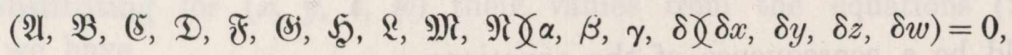

where $\mathfrak{A}, \ldots$ are the inverse system derived from the second differential coefficients of $\square:(\alpha, \beta, \gamma, \delta)$ are arbitrary coefficients, introduced only for symmetry, and there is no real loss of generality in reducing all but one of them to zero, and so reducing the equation for example to the form

$$
\mathfrak{R} \delta x+\mathfrak{S} \delta y+(5 \delta \delta+\mathfrak{\imath} \delta w=0 .
$$

The existence of the two linear equations between $(\delta x, \delta y, \delta z, \delta w)$ proves that $(x, y, z, w)$ are connected by a two-fold relation, that is, that the reciprocal of the given torse is a curve.

Cambridge, January 26, 1865. 